\title{
NOTE ON SOME RESULTS FOR ASYMPTOTICALLY PSEUDOCONTRACTIVE MAPPINGS AND ASYMPTOTICALLY NONEXPANSIVE MAPPINGS
}

YUCHAO TANG AND LIWEI LIU

Received 26 May 2006; Revised 5 August 2006; Accepted 13 August 2006

We discuss convergence theorems of modified Ishikawa and Mann iterative sequences with errors for asymptotically pseudocontractive and asymptotically nonexpansive mappings in Banach spaces, and the boundedness of the domain and range can be dropped, generalizing theorems of Chang.

Copyright (c) 2006 Y. Tang and L. Liu. This is an open access article distributed under the Creative Commons Attribution License, which permits unrestricted use, distribution, and reproduction in any medium, provided the original work is properly cited.

\section{Introduction and preliminaries}

Throughout this paper, we assume that $E$ is a real Banach space, $E^{*}$ is the topological dual space of $E,\langle\cdot, \cdot\rangle$ is the dual between $E$ and $E^{*}, D(T)$ and $F(T)$ denote the domain of $T$ and the set of all fixed points of $T$, respectively, and $J: E \rightarrow 2^{E^{*}}$ is the normalized duality mapping defined by

$$
J(x)=\left\{f \in E^{*}:\langle x, f\rangle=\|x\| \cdot\|f\|,\|f\|=\|x\|\right\}, \quad x \in E .
$$

Definition 1.1. Let $T: D(T) \subset E \rightarrow E$ be a mapping.

(1) $T$ is said to be asymptotically nonexpansive if there exists a sequence $\left\{k_{n}\right\}$ in $(0, \infty)$ with $\lim _{n \rightarrow \infty} k_{n}=1$ such that

$$
\left\|T^{n} x-T^{n} y\right\| \leq k_{n}\|x-y\|
$$

for all $x, y \in D(T)$ and $n=1,2, \ldots$

(2) $T$ is said to be asymptotically pseudocontractive if there exists a sequence $\left\{k_{n}\right\}$ in $(0, \infty)$ with $\lim _{n \rightarrow \infty} k_{n}=1$, and for any $x, y \in D(T)$ there exists $j(x-y) \in J(x-y)$ such that

$$
\left\langle T^{n} x-T^{n} y, j(x-y)\right\rangle \leq k_{n}\|x-y\|^{2}
$$

for all $n=1,2, \ldots$

Hindawi Publishing Corporation

Fixed Point Theory and Applications

Volume 2006, Article ID 24978, Pages 1-7

DOI 10.1155/FPTA/2006/24978 
2 Some results for asymptotically pseudocontractive mappings

(3) $T$ is said to be uniformly $L$-Lipschitzian if there exists $L>0$ such that

$$
\left\|T^{n} x-T^{n} y\right\| \leq L\|x-y\|
$$

for all $x, y \in D(T)$ and $n=1,2, \ldots$

The following proposition follows from Definition 1.1 immediately.

Proposition 1.2. (1) If $T: D(T) \subset E \rightarrow E$ is nonexpansive, then $T$ is an asymptocially nonexpansive mapping with a constant sequence $\{1\}$.

(2) If $T: D(T) \subset E \rightarrow E$ is asymptotically nonexpansive, then $T$ is a uniformly L-Lipschitzian, where $L=\sup _{n \geq 1}\left\{k_{n}\right\}$ and asymptotically pseudocontractive mapping.

Definition 1.3. (1) Let $T: D(T) \subset E \rightarrow E$ be a mapping, let $D(T)$ be a nonempty convex subset of $E$, let $x_{0} \in D(T)$ be a given point, and let $\left\{\alpha_{n}\right\},\left\{\beta_{n}\right\},\left\{\gamma_{n}\right\}$, and $\left\{\delta_{n}\right\}$ be four sequences in $[0,1]$. Then the sequence $\left\{x_{n}\right\}$ defined by

$$
\begin{aligned}
x_{n+1} & =\left(1-\alpha_{n}-\gamma_{n}\right) x_{n}+\alpha_{n} T^{n} y_{n}+\gamma_{n} u_{n}, \quad \forall n \geq 0, \\
y_{n} & =\left(1-\beta_{n}-\delta_{n}\right) x_{n}+\beta_{n} T^{n} x_{n}+\delta_{n} v_{n},
\end{aligned}
$$

is called the modified Ishikawa iterative sequence with errors of $T$, where $\left\{u_{n}\right\}$ and $\left\{v_{n}\right\}$ are two bounded sequences in $D(T)$.

(2) In (1.5) if $\beta_{n}=0$ and $\delta_{n}=0, n=0,1,2, \ldots$, then $y_{n}=x_{n}$. The sequence $\left\{x_{n}\right\}$ defined by

$$
x_{n+1}=\left(1-\alpha_{n}-\gamma_{n}\right) x_{n}+\alpha_{n} T^{n} x_{n}+\gamma_{n} u_{n}, \quad \forall n \geq 0,
$$

is called the modified Mann iterative sequence with errors of $T$.

In this paper, we discuss convergence theorems of modified Ishikawa and Mann iterative sequences with errors for asymptotically pseudocontractive and asymptotically nonexpansive mappings in Banach spaces, and the boundedness of the domain and range can be dropped, generalizing theorems of Chang [1].

Lemma $1.4[4]$. Let $\left\{A_{n}\right\},\left\{B_{n}\right\}$, and $\left\{C_{n}\right\}$ be sequences of nonnegative real numbers satisfying the inequality

$$
A_{n+1} \leq\left(1+B_{n}\right) A_{n}+C_{n}, \quad \forall n \geq 0 .
$$

If $\sum_{n=0}^{\infty} B_{n}<+\infty$ and $\sum_{n=0}^{\infty} C_{n}<+\infty$, then $\lim _{n \rightarrow \infty} A_{n}$ exists.

\section{Main results}

Lemma 2.1. Let $E$ be an arbitrary real Banach space, let $D$ be a nonempty closed convex subset of $E$, let $T: D \rightarrow D$ be a uniformly L-Lipschitzian asymptotically pseudocontractive mapping with a sequence $\left\{k_{n}\right\} \subset[1, \infty), \sum_{n=0}^{\infty}\left(k_{n}-1\right)<+\infty$. Let $\left\{\alpha_{n}\right\},\left\{\beta_{n}\right\},\left\{\gamma_{n}\right\}$, and 
$\left\{\delta_{n}\right\}$ be four sequences in $[0,1]$ satisfying the following conditions:

(i) $\alpha_{n}+\gamma_{n} \leq 1, \beta_{n}+\delta_{n} \leq 1$;

(ii) $\beta_{n} \leq \alpha_{n}, \delta_{n} \leq \gamma_{n}$, for all $n \geq 0$;

(iii) $\sum_{n=0}^{\infty} \gamma_{n}<+\infty$;

(iv) $\sum_{n=0}^{\infty} \alpha_{n}^{2}<+\infty$.

Let $x_{0} \in D$ be any given point and let $\left\{x_{n}\right\}$ and $\left\{y_{n}\right\}$ be the modified Ishikawa iterative sequence with errors defined by (1.5). If $F(T) \neq \varnothing$, then for any given $q \in F(T)$, $\lim _{n \rightarrow \infty}\left\|x_{n}-q\right\|$ exists.

Proof. Set $M=\max \left\{\sup _{n \geq 0}\left\|u_{n}-q\right\|, \sup _{n \geq 0}\left\|v_{n}-q\right\|\right\}$.

Since $T$ is asymptotically pseudocontractive, for all $x, y \in D$, there exists $j(x-y) \in$ $J(x-y)$ such that

$$
\left\langle T^{n} x-T^{n} y, j(x-y)\right\rangle \leq k_{n}\|x-y\|^{2} .
$$

Then from inequality (2.1), we obtain

$$
\left\langle\left(k_{n} I-T^{n}\right) x-\left(k_{n} I-T^{n}\right) y, j(x-y)\right\rangle=k_{n}\|x-y\|^{2}-\left\langle T^{n} x-T^{n} y, j(x-y)\right\rangle \geq 0,
$$

and it follows from Kato [2] that

$$
\|x-y\| \leq\left\|x-y+\lambda\left[\left(k_{n} I-T^{n}\right) x-\left(k_{n} I-T^{n}\right) y\right]\right\|, \quad \forall x, y \in D, \lambda>0 .
$$

Set $a_{n}:=\alpha_{n}+\gamma_{n}$. Then from the recursive formula (1.5), we have $x_{n+1}=\left(1-a_{n}\right) x_{n}+$ $a_{n} T^{n} y_{n}-y_{n}\left(T^{n} y_{n}-u_{n}\right)$. It follows that

$$
\begin{aligned}
x_{n}= & \left(1+a_{n}\right) x_{n+1}+a_{n}\left(k_{n} I-T^{n}\right) x_{n+1}-a_{n} k_{n} x_{n}+a_{n}^{2}\left(1+k_{n}\right)\left(x_{n}-T^{n} y_{n}\right) \\
& +a_{n}\left(T^{n} x_{n+1}-T^{n} y_{n}\right)+\gamma_{n}\left[1+a_{n}\left(1+k_{n}\right)\right]\left(T^{n} y_{n}-u_{n}\right) .
\end{aligned}
$$

Observe that

$$
q=\left(1+a_{n}\right) q+a_{n}\left(k_{n} I-T^{n}\right) q-a_{n} k_{n} q .
$$

So that

$$
\begin{aligned}
x_{n}-q= & \left(1+a_{n}\right)\left(x_{n+1}-q\right)+a_{n}\left[\left(k_{n} I-T^{n}\right) x_{n+1}-\left(k_{n} I-T^{n}\right) q\right]-a_{n} k_{n}\left(x_{n}-q\right) \\
& +a_{n}^{2}\left(1+k_{n}\right)\left(x_{n}-T^{n} y_{n}\right)+a_{n}\left(T^{n} x_{n+1}-T^{n} y_{n}\right)+\gamma_{n}\left[1+a_{n}\left(1+k_{n}\right)\right]\left(T^{n} y_{n}-u_{n}\right) .
\end{aligned}
$$


4 Some results for asymptotically pseudocontractive mappings

\section{Hence}

$$
\begin{aligned}
\left\|x_{n}-q\right\| \geq & \left(1+a_{n}\right)\left\|x_{n+1}-q+\frac{a_{n}}{1+a_{n}}\left[\left(k_{n} I-T^{n}\right) x_{n+1}-\left(k_{n} I-T^{n}\right) q\right]\right\|-a_{n} k_{n}\left\|x_{n}-q\right\| \\
& -a_{n}^{2}\left(1+k_{n}\right)\left\|x_{n}-T^{n} y_{n}\right\|-a_{n}\left\|T^{n} x_{n+1}-T^{n} y_{n}\right\| \\
& -\gamma_{n}\left[1+a_{n}\left(1+k_{n}\right)\right]\left\|T^{n} y_{n}-u_{n}\right\| \\
\geq & \left(1+a_{n}\right)\left\|x_{n+1}-q\right\|-a_{n} k_{n}\left\|x_{n}-q\right\|-a_{n}^{2}\left(1+k_{n}\right)\left\|x_{n}-T^{n} y_{n}\right\| \\
& -a_{n}\left\|T^{n} x_{n+1}-T^{n} y_{n}\right\|-\gamma_{n}\left[1+a_{n}\left(1+k_{n}\right)\right]\left\|T^{n} y_{n}-u_{n}\right\| .
\end{aligned}
$$

So

$$
\begin{aligned}
\left\|x_{n+1}-q\right\| \leq & {\left[1+\frac{a_{n}}{1+a_{n}}\left(k_{n}-1\right)\right]\left\|x_{n}-q\right\|+a_{n}^{2}\left(1+k_{n}\right)\left\|x_{n}-T^{n} y_{n}\right\| } \\
& +a_{n}\left\|T^{n} x_{n+1}-T^{n} y_{n}\right\|+\gamma_{n}\left[1+a_{n}\left(1+k_{n}\right)\right]\left\|T^{n} y_{n}-u_{n}\right\| .
\end{aligned}
$$

Furthermore, set $b_{n}:=\beta_{n}+\delta_{n}$. Then from recursive formula (1.5), we have $y_{n}=(1-$ $\left.b_{n}\right) x_{n}+b_{n} T^{n} x_{n}-\delta_{n}\left(T^{n} x_{n}-v_{n}\right)$. By condition (ii), we have $b_{n} \leq a_{n}$, for all $n \geq 0$. We make the following estimates:

$$
\begin{aligned}
\left\|y_{n}-q\right\| & =\left\|\left(1-b_{n}\right)\left(x_{n}-q\right)+b_{n}\left(T^{n} x_{n}-q\right)-\delta_{n}\left(T^{n} x_{n}-v_{n}\right)\right\| \\
\leq & {\left[1+b_{n}(L-1)\right]\left\|x_{n}-q\right\|+\delta_{n}\left\|T^{n} x_{n}-v_{n}\right\| } \\
\leq & L\left\|x_{n}-q\right\|+\delta_{n} L\left\|x_{n}-q\right\|+\delta_{n} M \\
= & L\left(1+\delta_{n}\right)\left\|x_{n}-q\right\|+\delta_{n} M, \\
\left\|x_{n}-T^{n} y_{n}\right\| \leq & \left\|x_{n}-q\right\|+L\left\|y_{n}-q\right\| \\
\leq & \left\|x_{n}-q\right\|+L^{2}\left(1+\delta_{n}\right)\left\|x_{n}-q\right\|+L \delta_{n} M \\
\leq & {\left[1+L^{2}\left(1+\delta_{n}\right)\right]\left\|x_{n}-q\right\|+L \delta_{n} M, } \\
\left\|T^{n} y_{n}-u_{n}\right\| \leq & L\left\|y_{n}-q\right\|+\left\|u_{n}-q\right\| \\
\leq & L^{2}\left(1+\delta_{n}\right)\left\|x_{n}-q\right\|+\left(1+L \delta_{n}\right) M, \\
\left\|T^{n} x_{n+1}-T^{n} y_{n}\right\| \leq & L\left\|x_{n+1}-y_{n}\right\| \\
= & L\left\|x_{n}-y_{n}+a_{n}\left(T^{n} y_{n}-x_{n}\right)-\gamma_{n}\left(T^{n} y_{n}-u_{n}\right)\right\| \\
\leq & L\left\|x_{n}-y_{n}\right\|+L a_{n}\left\|T^{n} y_{n}-x_{n}\right\|+L \gamma_{n}\left\|T^{n} y_{n}-u_{n}\right\| \\
= & L\left\|b_{n}\left(x_{n}-T^{n} x_{n}\right)+\delta_{n}\left(T^{n} x_{n}-v_{n}\right)\right\| \\
& +L a_{n}\left\|T^{n} y_{n}-x_{n}\right\|+L \gamma_{n}\left\|T^{n} y_{n}-u_{n}\right\|
\end{aligned}
$$




$$
\begin{aligned}
\leq & L b_{n}\left\|x_{n}-T^{n} x_{n}\right\|+L \delta_{n}\left\|T^{n} x_{n}-v_{n}\right\| \\
& +L a_{n}\left\|T^{n} y_{n}-x_{n}\right\|+L \gamma_{n}\left\|T^{n} y_{n}-u_{n}\right\| \\
\leq & L a_{n}(1+L)\left\|x_{n}-q\right\|+L^{2} \delta_{n}\left\|x_{n}-q\right\| \\
& +L \delta_{n} M+L a_{n}\left[1+L^{2}\left(1+\delta_{n}\right)\right]\left\|x_{n}-q\right\| \\
& +L^{2} a_{n} \delta_{n} M+L^{3} \gamma_{n}\left(1+\delta_{n}\right)\left\|x_{n}-q\right\|+L \gamma_{n}\left(1+L \delta_{n}\right) M .
\end{aligned}
$$

Using (2.9) and (2.10) in (2.8), we obtain the following estimation:

$$
\begin{aligned}
\left\|x_{n+1}-q\right\| \leq & {\left[1+\frac{a_{n}}{1+a_{n}}\left(k_{n}-1\right)\right]\left\|x_{n}-q\right\|+a_{n}^{2}\left(1+k_{n}\right)\left[1+L^{2}\left(1+\delta_{n}\right)\right]\left\|x_{n}-q\right\| } \\
& +a_{n}^{2}\left(1+k_{n}\right) L \delta_{n} M+L a_{n}^{2}(1+L)\left\|x_{n}-q\right\|+L^{2} a_{n} \delta_{n}\left\|x_{n}-q\right\|+L a_{n} \delta_{n} M \\
& +L a_{n}^{2}\left[1+L^{2}\left(1+\delta_{n}\right)\right]\left\|x_{n}-q\right\|+L^{2} a_{n}^{2} \delta_{n} M+L^{3} a_{n} \gamma_{n}\left(1+\delta_{n}\right)\left\|x_{n}-q\right\| \\
& +L a_{n} \gamma_{n}\left(1+L \delta_{n}\right) M+\gamma_{n}\left[1+a_{n}\left(1+k_{n}\right)\right] L^{2}\left(1+\delta_{n}\right)\left\|x_{n}-q\right\| \\
& +\gamma_{n}\left[1+a_{n}\left(1+k_{n}\right)\right]\left(1+L \delta_{n}\right) M \\
= & \left\{1+\frac{a_{n}}{1+a_{n}}\left(k_{n}-1\right)+a_{n}^{2}\left(1+k_{n}\right)\left[1+L^{2}\left(1+\delta_{n}\right)\right]+L a_{n}^{2}(1+L)+L^{2} a_{n} \delta_{n}\right. \\
& +L a_{n}^{2}\left[1+L^{2}\left(1+\delta_{n}\right)\right]+L^{3} a_{n} \gamma_{n}\left(1+\delta_{n}\right) \\
& \left.+\gamma_{n}\left[1+a_{n}\left(1+k_{n}\right)\right] L^{2}\left(1+\delta_{n}\right)\right\}\left\|x_{n}-q\right\| \\
& +\left\{a_{n}^{2}\left(1+k_{n}\right) L \delta_{n} M+L a_{n} \delta_{n} M+L^{2} a_{n}^{2} \delta_{n} M\right. \\
& \left.+L a_{n} \gamma_{n}\left(1+L \delta_{n}\right) M+\gamma_{n}\left[1+a_{n}\left(1+k_{n}\right)\right]\left(1+L \delta_{n}\right) M\right\} .
\end{aligned}
$$

Set

$$
\begin{aligned}
A_{n}:= & \left\|x_{n}-q\right\|, \\
B_{n}:= & \frac{a_{n}}{1+a_{n}}\left(k_{n}-1\right)+a_{n}^{2}\left(1+k_{n}\right)\left[1+L^{2}\left(1+\delta_{n}\right)\right]+L a_{n}^{2}(1+L)+L^{2} a_{n} \delta_{n} \\
& +L a_{n}^{2}\left[1+L^{2}\left(1+\delta_{n}\right)\right]+L^{3} a_{n} \gamma_{n}\left(1+\delta_{n}\right)+\gamma_{n}\left[1+a_{n}\left(1+k_{n}\right)\right] L^{2}\left(1+\delta_{n}\right), \\
C_{n}:= & a_{n}^{2}\left(1+k_{n}\right) L \delta_{n} M+L a_{n} \delta_{n} M+L^{2} a_{n}^{2} \delta_{n} M+L a_{n} \gamma_{n}\left(1+L \delta_{n}\right) M \\
& +\gamma_{n}\left[1+a_{n}\left(1+k_{n}\right)\right]\left(1+L \delta_{n}\right) M,
\end{aligned}
$$


6 Some results for asymptotically pseudocontractive mappings

then inequality (2.11) is equal to

$$
A_{n+1} \leq\left(1+B_{n}\right) A_{n}+C_{n}
$$

By conditions (ii), (iii), and (iv), we know $\sum_{n=0}^{\infty} B_{n}<+\infty, \sum_{n=0}^{\infty} C_{n}<+\infty$. By Lemma 1.4, we know $\lim _{n \rightarrow \infty}\left\|x_{n}-q\right\|$ exists.

Theorem 2.2. Let $E$ be a real uniformly smooth Banach space, let $D$ be a nonempty closed convex subset of $E$, and let $T: D \rightarrow D$ be a uniformly L-Lipschitzian asymptotically pseudocontractive mapping with a sequence $\left\{k_{n}\right\} \subset[1, \infty), \sum_{n=0}^{\infty}\left(k_{n}-1\right)<+\infty$. Let $\left\{\alpha_{n}\right\},\left\{\beta_{n}\right\}$, $\left\{\gamma_{n}\right\}$, and $\left\{\delta_{n}\right\}$ be four sequences in $[0,1]$ satisfying the conditions (i)-(iv) in Lemma 2.1.

Let $x_{0} \in D$ be any given point and let $\left\{x_{n}\right\},\left\{y_{n}\right\}$ be the modified Ishikawa iterative sequence with errors defined by (1.5).

(1) If $\left\{x_{n}\right\}$ converges strongly to a fixed point $q$ of $T$ in $D$, then there exists a nondecreasing function $\phi:[0, \infty) \rightarrow[0, \infty), \phi(0)=0$ such that

$$
\left\langle T^{n} y_{n}-q, J\left(y_{n}-q\right)\right\rangle \leq k_{n}\left\|y_{n}-q\right\|^{2}-\phi\left(\left\|y_{n}-q\right\|\right)
$$

for all $n \geq 0$.

(2) Conversely, if there exists a strictly increasing function $\phi:[0, \infty) \rightarrow[0, \infty), \phi(0)=0$ satisfying condition $(*)$, then $x_{n} \rightarrow q \in F(T)$.

Proof. Since $E$ is uniformly smooth, the normalized duality mapping $J: E \rightarrow E^{*}$ is singlevalued and uniformly continous on any bounded subset of $E$.

(1) Let $x_{n} \rightarrow q \in F(T)$. From conditions (ii)-(iv) in Lemma 2.1, we have $\beta_{n} \rightarrow 0, \delta_{n} \rightarrow 0$. Besides noticing $\left\|y_{n}-q\right\| \leq L\left(1+\delta_{n}\right)\left\|x_{n}-q\right\|+\delta_{n} M$ in (2.9) of Lemma 2.1, we have

$$
y_{n} \rightarrow q(n \longrightarrow \infty)
$$

The rest of the proof is the same as Chang's [1, Theorem 2.1].

(2) By Lemma 2.1, we know $\lim _{n \rightarrow \infty}\left\|x_{n}-q\right\|$ exists. So $\left\{x_{n}\right\}$ is bounded. And by the proof of Lemma 2.1, we can also get $\left\{T^{n} y_{n}-y_{n}\right\},\left\{x_{n}-T^{n} x_{n}\right\},\left\{x_{n}-v_{n}\right\}$, and $\left\{u_{n}-y_{n}\right\}$; all are bounded. So the rest of the proof is the same as Chang's [1, Theorem 2.1].

Remark 2.3. (1) Theorem 2.2 removes the restriction on $D$ which is bounded in Chang [1, Theorem 2.1].

(2) Respectively, we can get Chang [1, Theorems 2.2, 2.3, and 2.4], but without the restriction on $D$ which is bounded.

(3) In Osilike and Akuchu [3], they discussed the common fixed points of a family of asymptotically pseudocontractive maps. Our paper is different from it.

\section{Acknowledgments}

This project is supported by National Natural Science Foundations of China (10561007) and the Natural Science Foundations of JiangXi Province (0411036). 


\section{References}

[1] S. S. Chang, Some results for asymptotically pseudo-contractive mappings and asymptotically nonexpansive mappings, Proceedings of the American Mathematical Society 129 (2001), no. 3, 845853.

[2] T. Kato, Nonlinear semigroups and evolution equations, Journal of the Mathematical Society of Japan 19 (1967), 508-520.

[3] M. O. Osilike and B. G. Akuchu, Common fixed points of a finite family of asymptotically pseudocontractive maps, Fixed Point Theory and Applications 2004 (2004), no. 2, 81-88.

[4] K.-K. Tan and H. K. Xu, Approximating fixed points of nonexpansive mappings by the Ishikawa iteration process, Journal of Mathematical Analysis and Applications 178 (1993), no. 2, 301-308.

Yuchao Tang: Department of Mathematics, NanChang University, NanChang, JiangXi 330047, China E-mail address: hhaaoo1331@yahoo.com.cn

Liwei Liu: Department of Mathematics, NanChang University, NanChang, JiangXi 330047, China

E-mail address: liulws@yahoo.com.cn 